\title{
REMAINDERS OF COMPACTIFICATIONS AND THEIR RELATION TO A QUOTIENT LATTICE OF THE TOPOLOGY
}

\author{
G. D. FAULKNER AND M. C. VIPERA
}

(Communicated by Franklin D. Tall)

\begin{abstract}
If $\alpha X$ is a compactification of a locally compact space $X$, then the remainder associated with $\alpha X$ is the space $\alpha X \backslash X$. Frequently spaces which are quite different may have families of remainders which are related. This must be reflected somehow in the structure of the spaces. This paper studies one possible formulation of that similarity and its consequences.
\end{abstract}

\section{INTRODUCTION}

In what follows all topological spaces are assumed to be Hausdorff and locally compact except when specifically noted. If $X$ is a space, the set of all possible compactifications of $X$, up to equivalence, will be denoted by $\mathscr{K}(X)$. If $\alpha X$ is a compactification of $X$ then the remainder associated with $\alpha X$ is $\alpha X \backslash X$. The set of all remainders of a space $X$ will be denoted by $\mathscr{R}(X)$.

It is possible for nonhomeomorphic spaces $X$ and $Y$ that $\mathscr{R}(X)=\mathscr{R}(Y)$. This is clearly true if $\beta X \backslash X$ is homeomorphic to $\beta Y \backslash Y$, which may happen even if $X$ and $Y$ are quite different. For example, if $\kappa$ is a cardinal greater than $2^{c}$, then the Stone-Čech remainder of $Y=(\beta \mathbf{N} \backslash \mathbf{N}) \times \kappa$ is homeomorphic to $\beta \mathbf{N} \backslash \mathbf{N}$. Thus $\mathscr{R}(Y)=\mathscr{R}(\mathbf{N})$. In fact under the continuum hypothesis, if $X$ is a zero-dimensional, locally compact, $\sigma$-compact, noncompact space of weight no more than $c$, then $\beta X \backslash X$ and $\beta \mathbf{N} \backslash \mathbf{N}$ are homeomorphic.

It may also happen that $\mathscr{R}(X)=\mathscr{R}(Y)$ without $\beta X \backslash X \cong \beta Y \backslash Y$. This is the case if $X=\mathbf{R}^{+}=[0, \infty)$ and $Y=\mathbf{R}^{n}$ for $n \geq 2[2] . \quad \beta \mathbf{R}^{+} \backslash \mathbf{R}^{+}$is an indecomposable continua while, for example, $\beta \mathbf{R}^{2} \backslash \mathbf{R}^{2}$ is not.

The question we originally considered then was, given two spaces $X$ and $Y$, when is $\mathscr{R}(X)=\mathscr{R}(Y)$. We did not solve this problem, we only gave a sufficient condition which ensures $\beta X \backslash X \cong \beta Y \backslash Y$. The attempt to understand the problem led to other things.

How could the sets of remainders of two spaces coincide. The intuition is that as you go "out" in the spaces, they should appear related in some way. This paper attempts to make this intuition precise. In doing this we introduce a quotient lattice of the topology which reflects how the topology behaves off of compact sets. This lattice impacts on various aspects of the compactifications

Received by the editors April 1, 1992 and, in revised form, February 17, 1993.

1991 Mathematics Subject Classification. Primary 54D40, 54D35, 54D30. 
of the space and particularly on the Stone-Čech compactification. Somewhat unexpectedly it focuses attention on how properties such as normality are in some sense asymptotically determined by the topology.

\section{Preliminary Results}

Let $X$ be a locally compact space. We consider the following equivalence relation on the family $\mathscr{T}(X)$ of the open subsets of $X: U \sim V$ if the symmetric difference $U \Delta V$ is relatively compact. The quotient set $\mathscr{L}(X)$ is, in a natural way, a distributive lattice, because finite unions and intersections are compatible with that equivalence relation. We will denote by $[V]$ the equivalence class of $V$. Clearly $\mathscr{L}(X)$ captures the structure of the space as you "go out" in the space. Obviously $[X]$ and $[\varnothing]$ are the supremum and the infimum of $\mathscr{L}(X)$, respectively. General information on lattices can be found in [1].

Proposition 1.1. Let $[V] \in \mathscr{L}(X)$. Then [V] has a complement in $\mathscr{L}(X)$ if and only if its boundary $\operatorname{Fr}(V)$ is compact.

Proof. Let $\left[V^{\prime}\right]$ be the complement of $[V]$, so that $F=X \backslash\left(V \cup V^{\prime}\right)$ and $\overline{V \cap V^{\prime}}$ are compact. One has $\operatorname{Fr}(V) \subset V^{\prime} \cup F$, so we need only to prove that $\operatorname{Fr}(V) \cap V^{\prime}$ is relatively compact. Since $V^{\prime}$ is open, $\operatorname{Fr}(V) \cap V^{\prime} \subset \overline{V \cap V^{\prime}}$, so the conclusion follows. The converse is easy to prove.

We will denote by $\mathscr{E}(X)$ the set of the complemented elements of $\mathscr{L}(X)$. This set is a Boolean lattice (algebra).

Throughout this section we will denote by $A$ a distributive lattice with supremum 1 and infimum 0 . Let $a \in A$. We recall that $a^{\prime} \in A$ is said to be the pseudocomplement of $a$ if $a \wedge a^{\prime}=0$ and, for every $b \in A, b \wedge a=0$ implies $b \leq a$. If each $a \in A$ has a pseudocomplement, then $A$ is said to be pseudocomplemented. Obviously the pseudocomplement is unique.

We want to prove that $\mathscr{L}(X)$ is a pseudocomplemented lattice.

Proposition 1.2. Let $[U] \in \mathscr{L}(X)$. Then the class $[X \backslash \bar{U}]$ is the pseudocomplement of $[U]$.

Proof. Let $U^{\prime}=X \backslash \bar{U}=(X \backslash U)^{\circ}$. Clearly $[U] \wedge\left[U^{\prime}\right]=[\varnothing]$. If $[V] \in \mathscr{L}(X)$ satisfies $[U] \wedge[V]=[\varnothing]$, then there exists a compact $K \subset X$ such that $(V \backslash K) \cap$ $U=\varnothing$. Then $V \backslash K \subset(X \backslash U)^{\circ}=U^{\prime}$; hence $[V]=[V \backslash K] \leq\left[U^{\prime}\right]$.

For a lattice $A$, let $S(A)$ be the set of all ultrafilters in $A$. For $a \in A$ put

$$
h(a)=\{\mathscr{F} \in S(A) \mid a \in \mathscr{F}\} .
$$

It is easy to see that, for every $a, b \in A$, one has

$$
h(a) \cap h(b)=h(a \wedge b), \quad h(a) \cup h(b)=h(a \vee b) .
$$

Then the family $\{h(a) \mid a \in A\}$ is a basis for the closed subsets of a topology on $S(A)$. Under that topology $S(A)$ is a $T_{1}$-compact space.

It is well known that, if $A$ is a Boolean algebra, then $S(A)$ is a compact 0 -dimensional Hausdorff space and the Boolean algebra of its clopen subsets is isomorphic to $A$. Some of these properties are still true for pseudocomplemented lattices. 
Lemma 1.3. Let $A$ be a pseudocomplemented lattice. Then for every $a \in A$, $h(a)=S(A) \backslash h\left(a^{\prime}\right)$.

Proof. Clearly $h(a) \cap h\left(a^{\prime}\right)=\varnothing$. Let $\mathscr{F} \notin h(a)$, that is, $a \notin \mathscr{F}$. Then there is $b \in \mathscr{F}$ such that $a \wedge b=0$. By the definition of pseudocomplement, one has $b \leq a^{\prime}$ and this implies $a^{\prime} \in \mathscr{F}$, that is, $\mathscr{F} \in h\left(a^{\prime}\right)$.

Therefore one has

Proposition 1.4. Let $A$ be a pseudocomplemented lattice. Then $S(A)$ is a compact 0-dimensional Hausdorff space.

For any space $Y$, we will denote by $C O(Y)$ the Boolean algebra of the clopen subsets of $Y$.

Remark. If $A$ is pseudocomplemented, the mapping $h: A \rightarrow C O(S(A))$ is a surjective lattice homomorphism. Since $h(a)=h\left(a^{\prime \prime}\right), h$ injective implies $a=a^{\prime \prime}$ for every $a \in A$. This is true if and only if $A$ is a Boolean algebra.

For a pseudocomplemented lattice $A$, let $A^{\prime}=\left\{a^{\prime} \mid a \in A\right\}$. It is known that $A^{\prime}$ is a subsemilattice of $A$ with respect to the operation $\wedge$, but, in general, it is not a sublattice. However, $A^{\prime}$ is a Boolean algebra with respect to the operations $\wedge$ and $\vee^{\prime}$, where $a \vee^{\prime} b=\left(a^{\prime} \wedge b^{\prime}\right)^{\prime}$.

Proposition 1.5. If $A$ is a pseudocomplemented lattice, then the map $m: S(A)$ $\rightarrow S\left(A^{\prime}\right)$, defined by $m(\mathscr{F})=\mathscr{F} \cap A^{\prime}$, is a homeomorphism.

Proof. Let $\mathscr{F} \in S(A)$. First we will prove $\mathscr{F} \cap A^{\prime} \in S\left(A^{\prime}\right)$. Since the operation $\wedge$ is the same for $A$ and $A^{\prime}, \mathscr{F} \cap A^{\prime}$ is a proper filter. Suppose $\mathscr{G}$ is a filter on $A^{\prime}$ containing $\mathscr{F} \cap A^{\prime}$. Then $\mathscr{G}$ is a filter base on $A$. Let $\mathscr{H}$ be any ultrafilter on $A$ containing $\mathscr{G}$ and let $a \in \mathscr{F}$. Since, by Lemma $1.3, h(a)=h\left(a^{\prime \prime}\right)$, one has $a^{\prime \prime} \in \mathscr{F} \cap A^{\prime} \subset \mathscr{H}$. Thus $a \in \mathscr{H}$, so $\mathscr{F} \subset \mathscr{H}$, that is, $\mathscr{F}=\mathscr{H}$. This implies $\mathscr{F} \cap A^{\prime}=\mathscr{G}$, so $\mathscr{F} \cap A^{\prime}$ is maximal and $m$ is well defined.

To show continuity, we note that, for $a^{\prime} \in A^{\prime}$, one has $m^{-1}\left(h^{\prime}\left(a^{\prime}\right)\right)=h\left(a^{\prime}\right)$, where $h^{\prime}\left(a^{\prime}\right)$ denotes a basic open and closed subset of $S\left(A^{\prime}\right)$.

Let $\mathscr{G} \in S\left(A^{\prime}\right)$ and let $\mathscr{H}$ be an ultrafilter on $A$ containing $\mathscr{G}$. Clearly $m(\mathscr{H})=\mathscr{G}$, so that $m$ is surjective.

Let $\mathscr{F}, \mathscr{F}_{1} \in S(A)$, with $\mathscr{F} \neq \mathscr{F}_{1}$. Then there exist $a \in \mathscr{F}, b \in \mathscr{F}_{1}$ such that $a \wedge b=0$. Then one has $h\left(a^{\prime \prime}\right) \cap h\left(b^{\prime \prime}\right)=\varnothing$ and this implies $a^{\prime \prime} \wedge b^{\prime \prime}=0$. Since $a^{\prime \prime} \in \mathscr{F}$ and $b^{\prime \prime} \in \mathscr{F}_{1}, m(\mathscr{F})$ and $m\left(\mathscr{F}_{1}\right)$ contain disjoint elements. This proves that $m$ is injective.

Let $\mathscr{L}^{\prime}(X)$ be the set of pseudocomplements of elements of $\mathscr{L}(X)$. It is easy to see that $[U] \in \mathscr{L}^{\prime}(X)$ if and only if $[U]$ contains a regular open set.

Corollary 1.6. $S(\mathscr{L}(X))$ is a compact, 0-dimensional Hausdorff space and $S(\mathscr{L}(X)) \cong S\left(\mathscr{L}^{\prime}(X)\right)$.

Let $A$ be a lattice and let $M(A)$ be the set of the maximal ideals of $A$. For $a \in A$ put

Then one has

$$
d(a)=\{\mathscr{M} \in M(A) \mid a \notin \mathscr{M}\} \text {. }
$$

$$
d(a) \cap d(b)=d(a \wedge b), \quad d(a) \cup d(b)=d(a \vee b)
$$

The family $\{d(a) \mid a \in A\}$ is a basis for (the open subsets of) a topology on $M(A)$. It is known that $M(A)$, endowed with that topology, is a $T_{1}$-compact space. 
Proposition 1.7. If $X$ is a normal space, then $M(\mathscr{L}(X))$ is Hausdorff.

Proof. Let $\mathscr{M}_{1}, \mathscr{M}_{2}$ be distinct elements of $M(\mathscr{L}(X))$. Then there are $[U] \in$ $\mathscr{M}_{1}$ and $[V] \in \mathscr{M}_{2}$ such that $[U] \vee[V]=[X]$. Then $U \cup V=X \backslash K$, where $K$ is compact. We can replace $U$ by $U \cup W$, where $W$ is a relatively compact neighborhood of $K$, so we can suppose $U \cup V=X$. By normality, there are closed subsets $F \subset U, G \subset V$ such that $F \cup G=X$. Put $S=X \backslash F, T=X \backslash G$. One has $[U] \vee[S]=[X]$, so that $[S] \notin \mathscr{M}_{1}$, that is, $\mathscr{M}_{1} \in d([S])$. Similarly, $\mathscr{M}_{2} \in d([T])$. Since $S$ and $T$ are disjoint, one has $d([S]) \cap d([T])=\varnothing$.

\section{LATTICE HOMOMORPHISMS AND REMAINDERS}

In this section $X$ and $Y$ will be locally compact noncompact spaces.

Theorem 2.1. Let $X, Y$ be spaces and let $\rho: \mathscr{L}(X) \rightarrow \mathscr{L}(Y)$ be a lattice homomorphism satisfying

(a) $\rho([X])=[Y]$;

(b) $\rho([U])=[\varnothing]$ iff $[U]=[\varnothing]$

Then there is a map $r$ from $\mathscr{K}(X)$ to $\mathscr{K}(Y)$ which preserves the natural order and such that, for every $\alpha X \in \mathscr{K}(X), r(\alpha X)$ has the same remainder as $\alpha X$. In particular, $\mathscr{R}(X) \subset \mathscr{R}(Y)$.

Proof. Let $\alpha X \in \mathscr{K}(X)$ and let $K=\alpha X \backslash X$. For every open set $W$ in $\alpha X$, let $V_{W}=W \cap X, U_{W}=W \cap K$. Put, on the disjoint union $Y \cup K$, the topology generated by the family

$$
\mathscr{U}=\bigcup_{W}\left\{U_{W} \cup T \mid T \in \rho\left(\left[V_{W}\right]\right)\right\},
$$

which is closed with respect to finite intersections. The original topologies are induced on $K$ and on $Y$. In fact, for $W=\varnothing, \rho\left(\left[V_{W}\right]\right)=[\varnothing]$ is the set of all relatively compact open subsets of $Y$. We want to prove that $Y \cup K$, endowed with that topology, is a compactification of $Y$.

First, we will prove that the space is Hausdorff. Let $p, q \in Y \cup K, p \neq q$. If $p, q \in K$, then they have disjoint open neighborhoods $W, W^{\prime}$ in $\alpha X$. Then $\rho\left(\left[V_{W}\right]\right) \wedge \rho\left(\left[V_{W^{\prime}}\right]\right)=[\varnothing]$, hence there exist $T \in \rho\left(\left[V_{W}\right]\right), T^{\prime} \in \rho\left(\left[V_{W^{\prime}}\right]\right)$ with $T \cap T^{\prime}=\varnothing$. Then $U_{W} \cup T, U_{W^{\prime}} \cup T^{\prime}$ are disjoint open neighborhoods of $p$ and $q$ in $Y \cup K$. Now, suppose $p \in Y$ and let $U_{W} \cup T$ be any basic open neighborhood of $q$. Let $S$ be a relatively compact open neighborhood of $p$ in $Y$ such that $\bar{S}$ does not contain $q$. Then $U_{W} \cup(T \backslash \bar{S})$ is also a basic neighborhood of $q$ and it is disjoint from $S$.

To prove that $Y \cup K$ is compact, let $\left\{U_{W_{i}} \cup T_{i}\right\}$ be a basic open cover of $Y \cup K$. Then $\left\{U_{W_{i}}\right\}$ is a cover of $K$. Let $\left\{U_{W_{j}}\right\}$ be a finite subcover. This implies that $\left\{W_{j}\right\}$ covers all of $\alpha X$ except a compact set, hence $\bigvee_{j}\left[V_{W_{j}}\right]=[X]$. Therefore $\bigvee_{j} \rho\left(\left[V_{W_{j}}\right]\right)=[Y]$, that is, $\bigcup_{j} T_{j}$ covers all of $Y$ except a compact subset. So there exists a finite subcover of the given cover.

Finally $Y$ is dense in $Y \cup K$. Let $p \in K$ and let $U_{W} \cup T$ be a basic neighborhood of $p$. Since $p \in \mathrm{Cl}_{\alpha X}\left(V_{W}\right)$, one has $\left[V_{W}\right] \neq[\varnothing]$. Then $\rho\left(\left[V_{W}\right]\right) \neq[\varnothing]$ and this implies $T \neq \varnothing$.

Then $Y \cup K$ is a compactification of $Y$. We denote it by $\alpha_{1} Y$. If we put, for each $\alpha X \in \mathscr{K}(X), r(\alpha X)=\alpha_{1} Y$, we need only show that $r$ preserves the order. 
Suppose $\alpha X \leq \gamma X \in \mathscr{K}(X)$. Put $K_{1}=\gamma X \backslash X$. Let $q_{\gamma \alpha}: \gamma X \rightarrow \alpha X$ be the natural map and let $q: \gamma_{1} Y \rightarrow \alpha_{1} Y$ be defined by $\left.q\right|_{K_{1}}=\left.q_{\gamma \alpha}\right|_{K_{1}},\left.q\right|_{Y}=1_{Y}$. We wish to prove that $q$ is continuous. A basic open set in $\alpha_{1} Y$ is of the form $U_{W} \cup T$, where $W$ is an open subset of $\alpha X$ and $T \in \rho\left(\left[V_{W}\right]\right.$. We will prove that $q^{-1}\left(U_{W} \cup T\right)$ is open in $\gamma_{1} Y$. Let $W_{1}=q_{\gamma \alpha}^{-1}(W)$, which is open in $\gamma X$. One has $W_{1}=q_{\gamma_{\alpha}}^{-1}\left(U_{W}\right) \cup V_{W}$, so every set of the form $q_{\gamma \alpha}^{-1}\left(U_{W}\right) \cup S$, with $S \in \rho\left(\left[V_{W}\right]\right)$ is open in $\gamma_{1} Y$. But one has $q^{-1}\left(U_{W} \cup T\right)=q_{\gamma \alpha}^{-1}\left(U_{W}\right) \cup T$ and $T \in \rho\left(\left[V_{W}\right]\right)$, so $q^{-1}\left(U_{W} \cup T\right)$ is open, as we wanted.

We have proved that $r(\alpha X) \leq r(\gamma X)$.

Example. If $f: Y \rightarrow X$ is a dense compact map, then $[U] \mapsto\left[f^{-1}(U)\right]$ is a map from $\mathscr{L}(X)$ to $\mathscr{L}(Y)$ which satisfies the hypotheses of the above theorem, so one has $\mathscr{R}(X) \subset \mathscr{R}(Y)$. For example, let $f: \mathbf{R}^{n} \rightarrow \mathbf{R}^{+}$be given by $f(x)=|x|$. It follows that $\mathscr{R}\left(\mathbf{R}^{+}\right) \subset \mathscr{R}\left(\mathbf{R}^{n}\right)$, for each $n$.

For every open subset $V$ of $X$, put $\mathscr{L}_{V}=\{[U] \in \mathscr{L}(X) \mid[U] \leq[V]\}$. Suppose $F_{X}(V)$ is compact. It is easy to see that the map $\tau: \mathscr{L}(\bar{V}) \rightarrow \mathscr{L}(X)$ given by $[W] \mapsto[W \cap V]=\left[W \backslash F r_{X}(V)\right]$ is well defined and it is an injective lattice homomorphism whose image is $\mathscr{L}_{V}$. Therefore $\mathscr{L}(\bar{V}) \cong \mathscr{L}_{V}$. Furthermore it is easy to see that $\mathscr{R}(\bar{V}) \subset \mathscr{R}(X)$. This is trivial if $X \backslash V$ is compact. Otherwise we can consider its one-point compactification $(X \backslash V) \cup\{\infty\}$. Then, if $\alpha \bar{V}$ is a compactification of $\bar{V}$, whose remainder is $K$, we can identify $\infty$ with any point of $K$, so that we obtain a compactification of $X$ with remainder $K$. Therefore one has

Corollary 2.2. Let $X, Y$ be spaces and let $\eta: \mathscr{L}(X) \rightarrow \mathscr{L}(Y)$ be a lattice homomorphism such that $\eta([V])=[\varnothing]$ if and only if $[V]=[\varnothing]$. Suppose that $\eta([X])=[W]$, where $[W]$ is a complemented element of $\mathscr{L}(Y)$. Then $\mathscr{R}(X) \subset \mathscr{R}(Y)$.

Proof. One has $\operatorname{Im}(\eta) \subset \mathscr{L}_{W}$ which is isomorphic to $\mathscr{L}(\bar{W})$. Let $\rho: \mathscr{L}(X) \rightarrow$ $\mathscr{L}(\bar{W})$ be the composition of $\eta$ and that isomorphism. It is easy to see that $\rho$ satisfies all the hypotheses of Theorem 2.1 , so $\mathscr{R}(X) \subset \mathscr{R}(\bar{W})$. Since $W$ has compact boundary (see Proposition 1.1), by the above observation one has $\mathscr{R}(\bar{W}) \subset \mathscr{R}(Y)$, so the conclusion follows.

For $p \in X$, we denote by $\mathscr{N}_{X}(p)$ the set of the open neighborhoods of $p$. We recall that the singular set $\mathscr{S}(f)$ of a continuous map $f: Y \rightarrow X$ is defined to be $\left\{p \in X \mid \forall U \in \mathscr{N}_{X}(p), f^{-1}(U)\right.$ is not relatively compact $\}$. It is easy to see that, if $K \subset X$ is compact and $K \cap \mathscr{S}(f)=\varnothing$, then $f^{-1}(K)$ is compact.

Corollary 2.3. Let $f: Y \rightarrow X$ be a dense continuous map such that $\mathscr{S}(f)$ is compact. Then $\mathscr{R}(X) \subset \mathscr{R}(Y)$.

Proof. Let $U$ be a relatively compact neighborhood of the singular set $\mathscr{S}(f)$. Then $F r_{X}(X \backslash \bar{U})=F r_{X}(\bar{U})$ is compact and disjoint from $\mathscr{S}(f)$. Then $f^{-1}(X \backslash \bar{U})$ is an open subset of $X$ whose boundary is compact. If we put, for every open subset $V$ of $X, \eta([V])=\left[f^{-1}(V \backslash \bar{U})\right]$, then $\eta$ is well defined and satisfies the hypotheses of Corollary 2.2.

By Theorem 2.1, $\mathscr{L}(X) \cong \mathscr{L}(Y)$ implies $\mathscr{R}(X)=\mathscr{R}(Y)$. In fact more is true. 
Theorem 2.4. If $\mathscr{L}(X) \cong \mathscr{L}(Y)$, then $\beta X \backslash X \cong \beta Y \backslash Y$.

Proof. Let $\rho: \mathscr{L}(X) \rightarrow \mathscr{L}(Y)$ be an isomorphism and let $r: \mathscr{K}(X) \rightarrow \mathscr{K}(Y)$ be the map defined in the proof of Theorem 2.1. We want to show that $r$ is bijective. Since $\rho$ is an isomorphism, so is $\rho^{-1}$. Thus we can define $s: \mathscr{K}(Y) \rightarrow \mathscr{K}(X)$ in the same manner as $r$. We want to prove that sor $=1_{\mathscr{K}(X)}$. Let $\alpha X \in \mathscr{K}(X)$. We will prove that it is equivalent to $s(r(\alpha X))$. Since they have the same remainder, we need only prove that the set-theoretic identity from $s(r(\alpha X))$ to $\alpha X$ is continuous. Put $K=\alpha X \backslash X$ and let $W$ be any open subset of $\alpha X$. We will prove that $W$ is open in $s(r(\alpha X))$. By definition, if $T \in \rho\left(\left[V_{W}\right]\right)$, then $W^{\prime}=U_{W} \cup T$ is an open subset of $r(\alpha X)$. Then, by the definition of $s$, every set of the form $U_{W} \cup S$, with $S \in \rho^{-1}([T])$, is open in $s(r(\alpha X))$. One has $V_{W} \in\left[V_{W}\right]=\rho^{-1}\left(\rho\left(\left[V_{W}\right]\right)\right)=\rho^{-1}([T])$, so $U_{W} \cup V_{W}=W$ is open in $s(r(\alpha X))$, as we wanted. In the same way we can prove that $r \circ s=1_{\mathscr{R}(Y)}$.

We know that $r$ and $s$ preserve the order. Therefore $\mathscr{K}(X) \cong \mathscr{K}(Y)$, hence, by a theorem of Magill's [6], $\beta X \backslash X \cong \beta Y \backslash Y$.

The last theorem suggests we investigate relationships between $\mathscr{L}(X)$ and $\beta X \backslash X$.

We will say that the space $X$ satisfies the condition (C) if

Every nonrelatively compact subset of $X$ contains a closed noncompact subset of $X$.

Realcompact spaces satisfy (C). In fact, if $S \subset X$ is not relatively compact, then $\bar{S}$ is not pseudocompact. If $f: \bar{S} \rightarrow \mathbf{R}$ is unbounded, then it is unbounded on $S$. If we take, $\forall n \in \mathbf{N}$, a point $x_{n} \in S$ such that $\left|f\left(x_{n}\right)\right|>n$, then the set $\left\{x_{n}\right\}_{n \in \mathbf{N}}$ is infinite and has no accumulation point in $\bar{S}$, so it is closed in $X$ and noncompact.

Paracompact spaces also satisfy (C). If we assume that there are no measurable cardinals, then this is a consequence of the previous statement. However the same proof will yield the result for paracompact spaces without any assumptions.

Proposition 2.5. If $X$ is normal and satisfies condition (C) then the lattice $\mathscr{L}(X)$ is isomorphic to a basis for the open subsets of $\beta X \backslash X$.

Proof. Put $K=\beta X \backslash X, \mathscr{B}=\left\{K \backslash \mathrm{Cl}_{\beta X}(F) \mid F\right.$ is a closed subset of $\left.X\right\} . \mathscr{B}$ is a basis for the open subsets of $K$ which is closed with respect to finite unions and intersections. If $F, G$ are closed subsets of $X$, then $\mathrm{Cl}_{\beta X}(F) \cap K=$ $\mathrm{Cl}_{\beta X}(G) \cap K$ implies $F \Delta G$ is relatively compact. Otherwise it would contain a closed noncompact subset $H$, so that $\varnothing \neq \mathrm{Cl}_{\beta X}(H) \cap K \subset \mathrm{Cl}_{\beta X}(F \cap G) \cap K$, a contradiction. Then the map $\psi: K \backslash \mathrm{Cl}_{\beta X}(F) \mapsto[X \backslash F]$ is well defined and it is a lattice homomorphism from $\mathscr{B}$ onto $\mathscr{L}(X)$. It is clear that $\psi$ is bijective. In fact the map $[V] \mapsto K \backslash \mathrm{Cl}_{\beta X}(X \backslash V)$ is the inverse of $\psi$.

Example. For $X=\left[0, \omega_{1}\right), \mathscr{L}(X)$ has more than two elements. In fact, if $D$ is the set of nonisolated points, then $[X] \neq[X \backslash D] \neq[\varnothing]$. So in the above proposition it is not sufficient to suppose that $X$ is normal.

Let $K$ be a compact space and let $\mathscr{B}$ be an (open) basis of $K$, closed with respect to finite unions and intersections. Suppose that $\psi: \mathscr{B} \rightarrow \mathscr{L}(X)$ is an 
injective homomorphism, where $X$ is any (locally compact) space. If we put, on the disjoint union $X \cup K$, the topology generated by the family

$$
\bigcup_{U \in \mathscr{B}}\{U \cup V \mid V \in \psi(U)\},
$$

then we obtain a compactification of $X$ (with remainder $K$ ). We will denote it by $X \cup_{\psi} K$. The proof can essentially be found in [3].

Proposition 2.6. Let $K$ be a compact space and let $\mathscr{B}$ be a basis for the open subsets of $K$ which is closed with respect to finite unions and intersections. Let $X$ be any (locally compact) space. Suppose that there is a lattice isomorphism $\psi$ from $\mathscr{B}$ to $\mathscr{L}(X)$. Then $\beta X \backslash X \cong K$. Furthermore $X$ is normal.

Proof. The hypotheses imply that we can construct the compactification $X \cup_{\psi} K$ (see above). We will prove that it is equivalent to $\beta X$. Suppose that $F$ and $G$ are disjoint closed subsets of $X$ and let $V=X \backslash F, W=X \backslash G$. Then $[V] \vee[W]=[X]$. If $[V]=\psi\left(U_{1}\right),[W]=\psi\left(U_{2}\right)$, one has $U_{1} \cup U_{2}=K$, so $U_{1} \cup V$ and $U_{2} \cup W$ are open subsets of $X \cup_{\psi} K$ whose union is $X \cup_{\psi} K$. Their complements are disjoint closed subsets of $X \cup_{\psi} K$ which contain $F$ and $G$ respectively. This implies that $X \cup_{\psi} K=\beta X$ and $X$ is normal.

From this we have the following corollary which, surprisingly, shows that normality is somehow connected to the structure of $\mathscr{L}(X)$.

Corollary 2.7. Let $X, Y$ be spaces such that $\mathscr{L}(X) \cong \mathscr{L}(Y)$. Then if $X$ is normal and satisfies $(\mathrm{C})$, then $Y$ is also normal.

Example. Let $X=\beta \mathbf{R} \backslash(\beta \mathbf{N} \backslash \mathbf{N})$. Then $\beta X \backslash X$ is homeomorphic to $\beta \mathbf{N} \backslash \mathbf{N}$, but $\mathscr{L}(\mathbf{N})$ is not isomorphic to $\mathscr{L}(X)$ since $X$ is not normal (see the above corollary). Therefore the converse of Theorem 2.4 does not hold.

Remark. Let $\omega X$ be the Wallman extension of $X$ (that is, the space of the ultrafilters of closed subsets of $X$ ). Recall that $\omega X$ is Hausdorff if and only if $X$ is normal. In this case one has $\omega X=\beta X$. The proof of Proposition 2.5 also proves the following statement: if $X$ satisfies condition $(\mathrm{C})$ then $\mathscr{L}(X)$ is isomorphic to a basis for the open subsets of $\omega X \backslash X$. Therefore Proposition 2.6 implies that, if $X$ satisfies (C) and $\omega X \backslash X$ is Hausdorff, then $X$ is normal.

Now, in Proposition 2.6, let us suppose $K$ is a $T_{1}$-compact (not necessarily Hausdorff) space. The set $\mathscr{C}$ of the complements of the elements of $\mathscr{B}$ is a basis for the closed sets of $K$ which is also a lattice. Then we can prove that $K$ is homeomorphic to the space $S(\mathscr{C})$ of the ultrafilters of $\mathscr{C}$. Let us denote by $\mathscr{L}_{C}(X)$ the lattice of the equivalence classes of closed subsets of $X$ with respect to the relation $F \approx G$ if $F \Delta G$ is relatively compact. Since $[U]=[V]$ in $\mathscr{L}(X)$ implies $X \backslash U \approx X \backslash V$, the lattices $\mathscr{L}(X)$ and $\mathscr{L}_{C}(X)$ are isomorphic up to exchange of the operations. So one has $\mathscr{C} \cong \mathscr{L}_{C}(X)$ and $K \cong S(\mathscr{C}) \cong S\left(\mathscr{L}_{C}(X)\right)$. We can also prove that, for any space $X, S\left(\mathscr{L}_{C}(X)\right)$ is isomorphic to the space of the free ultrafilters of closed subsets of $X$ (to prove that we can use the same kind of arguments as in Theorem 3.5, see the next section). Then, with the same hypotheses as in Proposition 2.6, except that $K$ is assumed to be $T_{1}$ instead of $T_{2}$, we obtain $K \cong \omega X \backslash X$.

Since, clearly, $M(\mathscr{L}(X)) \cong S\left(\mathscr{L}_{C}(X)\right)$, we have also proved that, for each $X, M(\mathscr{L}(X)) \cong \omega X \backslash X$ (this provides another proof of Proposition 1.7). In 
particular, if $X$ is normal, one has $M(\mathscr{L}(X)) \cong \beta X \backslash X$. In the next theorem we will give a direct proof of this last statement, which does not use Wallman extensions.

Theorem 2.8. If $X$ is normal, then $\beta X \backslash X \cong M(\mathscr{L}(X))$.

Proof. Set $Z=X \cup M(\mathscr{L}(X))$ and put on $Z$ the topology generated by the family $\{U \cup d([U]) \mid U \in \mathscr{T}(X)\}$, which is closed with respect to finite intersections (see $\S 1$ ). If $U$ is relatively compact, then $d([U])=\varnothing$, so that all open subsets of $X$ are open in $Z$. Then $X$ is an open and $K$ is a closed subspace of $Z$. Furthermore $X$ is dense in $Z$.

Since $M(\mathscr{L}(X))$ is Hausdorff (see Proposition 1.7), we can show that $Z$ is also Hausdorff, using the same kind of arguments as in the proof of Theorem 2.1. Since $M(\mathscr{L}(X))$ is compact, it is easy to see that $Z$ is also compact. In fact, if $\bigcup_{i=1}^{n} d\left(\left[U_{i}\right]\right)=M(\mathscr{L}(X))$, then $\bigvee_{i=1}^{n}\left[U_{i}\right]=[X]$. Then $\left\{U_{i} \cup d\left(\left[U_{i}\right]\right)\right\}_{i=1}^{n}$ covers all of $Z$ except a compact set.

To prove that $Z=\beta X$ we need only show that, if $F, G$ are disjoint closed subsets of $X$, then they have disjoint closures in $Z$. Put $U=X \backslash F, V=$ $X \backslash G$. Then $U \cup V=X$, hence $(U \cup d([U])) \cup(V \cup d([V]))=Z$, so that $Z \backslash(U \cup d([U]))$ and $Z \backslash(V \cup d([V]))$ are closed subsets of $Z$ containing $F$ and $G$ respectively.

\section{STONE-ČECH REMAINDERS AND SPACES OF ULTRAFILTERS}

In this section we will study relationships between the Stone-Čech remainder of a locally compact space $X$ and the space of ultrafilters $S(\mathscr{L}(X))$. Sometimes they are clearly homeomorphic. It is well known that $\beta \mathbf{N}$ is homeomorphic to the space of the ultrafilters of the lattice $\mathscr{L}(\mathbf{N})$ of all subsets of $\mathbf{N}$ up to finite subsets. Clearly $\mathscr{L}(\mathbf{N})$ is Boolean, that is, $\mathscr{L}(\mathbf{N})=\mathscr{E}(\mathbf{N})$.

The condition $\mathscr{L}(X)=\mathscr{E}(X)$ is equivalent, by Proposition 1.1, to the property that all nowhere dense closed subsets of $X$ are compact. All spaces such that the set $D(X)$ of nonisolated points is compact have this property. In fact all nowhere dense closed subsets must be contained in $D(X)$. Katětov has shown [5] that a topological space without isolated points in which all nowhere dense subsets are compact must itself be compact.

Example. Let $A$ be a proper infinite closed subset of $\beta \mathbf{N} \backslash \mathbf{N}$. If $X=\mathbf{N} \cup A$ with the induced topology, then $D(X) \subseteq A$ is compact, hence $\mathscr{L}(X)=\mathscr{E}(X)$. Even if $A$ is taken to be a single point the family of such spaces includes $2^{c}$ homeomorphism classes.

Also, one has

Proposition 3.1. Suppose $X$ satisfies $\mathscr{L}(X)=\mathscr{E}(X)$. Then $D(X)$ is countably compact. Moreover, if $D(X)$ has no relatively isolated points, then it is compact. Proof. Suppose $A=\left\{a_{1}, a_{2}, \ldots\right\}$ is a countably infinite subset of $D(X)$ without accumulation points. Thus $A$ is closed in $X$. By hypothesis every open set in $X$ has compact boundary, hence $\operatorname{Fr}(X \backslash A)$ is compact. Thus there is $a_{i} \in A$ such that $a_{i} \notin F r(X \backslash A)$. Therefore $a_{i}$ must be isolated in $X$, a contradiction. The remainder follows from the theorem of Katětov mentioned above.

Now we need some results about compactifications with 0 -dimensional remainders. 
For any space $X$ we will denote by $\phi X$ the Freudenthal compactification of $X$. Then one has

Proposition 3.2. For any space $X$ there is a natural isomorphism $\mu_{\phi}: C O(\phi X \backslash X)$ $\rightarrow \mathscr{E}(X)$.

Proof. For each compactification $\alpha X$ such that $K=\alpha X \backslash X$ is 0-dimensional, we define a 1-1 homomorphism $\mu_{\alpha}: C O(K) \rightarrow \mathscr{L}(X)$ such that $X \cup_{\mu_{\alpha}} K$ is equivalent to $\alpha X$ (see $\S 2$ for the definition of $X \cup_{\mu_{\alpha}} K$ ). For $U \in C O(K)$ let $U^{\prime}=K \backslash U$. Let $W, W^{\prime}$ be disjoint open subsets of $\alpha X$ such that $W \cap K=U$, $W^{\prime} \cap K=U^{\prime}$. Let $V=W \cap X, V^{\prime}=W^{\prime} \cap X$. Note that $X \backslash\left(V \cup V^{\prime}\right)$ is compact, hence $\left[V^{\prime}\right]$ is the complement of $[V]$ in $\mathscr{L}(X)$. The closed set $F=\alpha X \backslash W$ satisfies $F \cap K \subset F^{\circ}$. Thus the class $[V]$ is independent on the choice of $W$ (see [3, proof of Theorem 4]). We put $\mu_{\alpha}(U)=[V]$. It is easy to prove that $\mu_{\alpha}$ is a lattice homomorphism, with $\mu_{\alpha}(K)=[X]$, and $\mathscr{E}_{\alpha}=\operatorname{Im}\left(\mu_{\alpha}\right)$ is a Boolean subalgebra of $\mathscr{E}(X)$. Furthermore $\mu_{\alpha}(U)=[\varnothing]$ if and only if $U=\varnothing$ and this implies that $\mu_{\alpha}$ is injective. To prove that $X \cup_{\mu_{\alpha}} K$ is equivalent to $\alpha X$ it is sufficient to observe that, by the definition of $\mu_{\alpha}$, the subsets of the form $U \cup T, T \in \mu_{\alpha}(U)$, which are the basic open subsets of $X \cup_{\mu_{\alpha}} K$, are also open in $\alpha X$.

On the other hand, if $\mathscr{E}$ is a Boolean subalgebra of $\mathscr{E}(X)$, let $K$ be a 0-dimensional compact space such that $C O(K)$ is isomorphic to $\mathscr{E}$ (that is, $K \cong S(\mathscr{E}))$. Let $\psi: C O(K) \rightarrow \mathscr{L}(X)$ be the composition of that isomorphism and the inclusion map and put $\alpha X=X \cup_{\psi} K$. Then one has $\mathscr{E}_{\alpha}=\mathscr{E}$. So the correspondence $\alpha X \mapsto \mathscr{E}_{\alpha}$ is a bijection from the set of the compactifications of $X$ with 0-dimensional remainder to the set of the Boolean subalgebras of $\mathscr{E}(X)$. We want to prove that $\mathscr{E}_{\phi}=\mathscr{E}(X)$. To do that it is sufficient to show that $\mathscr{E}_{\alpha} \subset \mathscr{E}_{\gamma}$ implies $\alpha X \leq \gamma X$. (Actually the converse is also true.) If $K$ and $K_{1}$ are the remainders of $\alpha X$ and $\gamma X$, respectively, we can put $\alpha X=$ $X \cup_{\mu_{\alpha}} K, \gamma X=X \cup_{\mu_{\gamma}} K_{1}$. Since $p=\mu_{\gamma}^{-1} \circ j \circ \mu_{\alpha}: C O(K) \rightarrow C O\left(K_{1}\right)$ is a 1-1 homomorphism, there is a surjective continuous function $p^{*}: K_{1} \rightarrow K$ such that $p^{*-1}(U)=p(U), \forall U \in C O(K)$. Let us define $q: \gamma X \rightarrow \alpha X$ to be the identity map on $X$ and to be equal to $p^{*}$ on the remainder $K_{1}$. We need only prove that $q$ is continuous. Let $U \cup V$, with $U \in C O(K)$ and $V \in \mu_{\alpha}(U)$, be a basic open subset of $\alpha X$. One has $q^{-1}(U \cup V)=p^{*-1}(U) \cup V=p(U) \cup V$. Since, by definition of $p, \mu_{\gamma}(p(U))=\mu_{\alpha}(U)=[V], q^{-1}(U \cup V)$ is a basic open set of $\gamma X$.

Corollary 3.3. If $\mathscr{L}(X)=\mathscr{E}(X)$, then $S(\mathscr{L}(X)) \cong \beta X \backslash X$.

Proof. By the above proposition, $C O(\phi X \backslash X) \cong \mathscr{L}(X)$, hence, by Proposition 2.6, $\beta X \backslash X \cong \phi X \backslash X$ (that is, $\beta X=\phi X)$. Then $S(\mathscr{L}(X))=S(\mathscr{E}(X)) \cong$ $S(C O(\phi X \backslash X)) \cong \phi X \backslash X=\beta X \backslash X$.

From the proof of Proposition 3.2 we can also deduce some results about 0 -dimensional remainders. We denote by $\mathscr{R}_{0}(X)$ the set of the 0 -dimensional remainders of $X$ (up to homeomorphism).

Proposition 3.4. Let $X$ be a space and let $K$ be a 0 -dimensional compact space. Then $K$ is a remainder of $X$ if and only if $C O(K)$ is isomorphic to a Boolean subalgebra of $\mathscr{E}(X)$. If $Y$ is another space, then $\mathscr{R}_{0}(X) \subset \mathscr{R}_{0}(Y)$ if and only if $\mathscr{E}(X)$ is isomorphic to a Boolean subalgebra of $\mathscr{E}(Y)$. 
Now we want to prove that, for any space $X, \beta X \backslash X$ is a continuous image of $S(\mathscr{L}(X))$.

We denote, as usual, by $E X$ the absolute of $X$, that is, the set of the convergent ultrafilters in $\mathscr{T}(X)$. It is known that $\beta E X$ can be identified with the Gleason space $S(\mathscr{T}(X))$. The family $\{h(U) \mid U \in \mathscr{T}(X)\}$ is a clopen basis for $S(\mathscr{T}(X)$ ) (in fact $\mathscr{T}(X)$ is a pseudocomplemented lattice, see $\S 1$ ). Information on absolutes and related results can be found in abundance in [7].

Theorem 3.5. For any space $X$, there exists a natural homeomorphism $p_{X}$ : $S(\mathscr{L}(X)) \rightarrow \beta E X \backslash E X$.

Proof. For each $F \in S(\mathscr{L}(X))$ let $\mathscr{F}_{F}$ denote the collection

$$
\mathscr{F}_{F}=\{V \in \mathscr{T}(X) \mid[V] \in F\} .
$$

Clearly $\mathscr{F}_{F}$ is a filter in $\mathscr{T}(X)$ and we will show that it is maximal. Let $V_{0} \in \mathscr{T}(X)$ such that $V_{0} \cap V \neq \varnothing$ for every $V \in \mathscr{F}$. This implies that $V_{0} \cap V$ cannot be relatively compact, otherwise $V \backslash \overline{V_{0} \cap V}$, which is equivalent to $V$, would be an element of $\mathscr{F}_{F}$ disjoint from $V_{0}$. Then $\left[V_{0}\right] \wedge[V] \neq[\varnothing]$ for each $[V] \in F$ and this implies $\left[V_{0}\right] \in F$, that is, $V_{0} \in \mathscr{F}_{F}$. Thus $\mathscr{F}_{F} \in \beta E X$. But it is clear that $\mathscr{F}_{F}$ cannot be convergent, otherwise it would contain some relatively compact open subsets of $X$, that is, $[\varnothing] \in F$. Then $\mathscr{F}_{F} \in \beta E X \backslash E X$ and we put $p_{X}(F)=\mathscr{F}$.

The map $p_{X}$ is injective. If $F_{1}, F_{2}$ are distinct elements of $S(\mathscr{L}(X))$. Then there exist $\left[V_{1}\right] \in F_{1},\left[V_{2}\right] \in F_{2}$ with $\left[V_{1}\right] \wedge\left[V_{2}\right]=[\varnothing]$. This means that there exist disjoint open sets $U_{1}, U_{2}$, equivalent to $V_{1}, V_{2}$, respectively. But one has $U_{1} \in p_{X}\left(F_{1}\right), U_{2} \in p_{X}\left(F_{2}\right)$, so that $p_{X}\left(F_{1}\right) \neq p_{X}\left(F_{2}\right)$.

Now let $\mathscr{F} \in \beta E X \backslash E X$. The set $F=\{[V] \in \mathscr{L}(X) \mid V \in \mathscr{F}\}$ is closed with respect to finite intersection and does not contain $[\varnothing]$. In fact, if $\mathscr{F}$ contained a relatively compact set, it would be convergent. Then $F$ is a filter base, so it is contained in an ultrafilter whose image must be $\mathscr{F}$. (Actually one can prove that $F$ is an ultrafilter.) We have shown that $p_{X}$ is surjective.

Since $S(\mathscr{L}(X))$ is compact, we will be done if we show that $p_{X}$ is continuous. But it is easy to see that, for each $U \in \mathscr{T}(X)$, one has

$$
p_{X}^{-1}(h(U) \cap(\beta E X \backslash E X))=h([U]) .
$$

This completes the proof.

Corollary 3.6. For any space $X$, there exists a surjective continuous function $g_{X}: S(\mathscr{L}(X)) \rightarrow \beta X \backslash X$.

Proof. We can take $g_{X}=l_{X} \circ p_{X}$, where $l_{X}: \beta E X \backslash E X \rightarrow \beta X \backslash X$ is the natural map which takes each ultrafilter to its limit point.

Now we want to give an equivalent condition for $g_{X}$ to be injective. Of course, if this is the case, $g_{X}$ is a homeomorphism and $\beta X \backslash X$ is 0 -dimensional. This is also equivalent to $l_{X}$ being a homeomorphism.

We recall that $\mathscr{L}^{\prime}(X)$, the set of pseudocomplemented elements of $\mathscr{L}(X)$, is a Boolean algebra with respect to the operations $\wedge$ and $\vee^{\prime}$ (see $\S 1$ ) and, by Proposition 1.5, there is a natural homeomorphism $m: S(\mathscr{L}(X)) \rightarrow S\left(\mathscr{L}^{\prime}(X)\right)$. Clearly $\mathscr{E}(X)$ is a subalgebra of $\mathscr{L}^{\prime}(X)$. We note that the operations $\vee$ and $\mathrm{V}^{\prime}$ coincide on $\mathscr{E}(X)$. 
It is known that, if $f: A \rightarrow B$ is a homomorphism of Boolean algebras then the map $f^{*}: S(B) \rightarrow S(A)$, defined by $f^{*}(\mathscr{F})=f^{-1}(\mathscr{F})$ is continuous and it is bijective (hence a homeomorphism) if and only if $f$ is an isomorphism. So, if we put $K=\phi X \backslash X$, then, by Proposition 3.1, we have a homeomorphism $\mu_{\phi}^{*}: S(\mathscr{E}(X)) \rightarrow S(C O(K))$. We can identify $S(C O(K))$ and $K$, that is, we identify each ultrafilter of clopen subsets of $K$ with its limit point. So one has, for $G \in S(\mathscr{E}(X)), \mu_{\phi}^{*}(G)=y$, where $\{y\}=\bigcap\left\{U \in C O(K) \mid \mu_{\phi}(U) \in G\right\}$.

Lemma 3.7. Let $j: \mathscr{E}(X) \rightarrow \mathscr{L}^{\prime}(X)$ be the inclusion map and let $t=j^{*} \circ$ $m: S(\mathscr{L}(X)) \rightarrow S(\mathscr{E}(X))$. Let $q: \beta X \backslash X \rightarrow \phi X \backslash X$ be the restriction of the natural map. Then the diagram

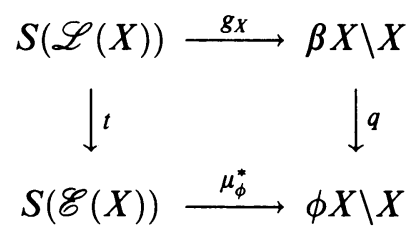

commutes.

Proof. Let $K=\phi X \backslash X$. For $F \in S(\mathscr{L}(X))$, put $p=g_{X}(F)$ and $z=q(p)$. Let $G=t(F)=F \cap \mathscr{E}(X)$ and $y=\mu_{\phi}^{*}(G)$. We need to prove $y=z$. One has

$$
\{p\}=\bigcap_{[V] \in F} \mathrm{Cl}_{\beta X}(V) \subset \bigcap_{[T] \in G} \mathrm{Cl}_{\beta X}(T) .
$$

This implies $z \in \bigcap_{[T] \in G} \mathrm{Cl}_{\phi X}(T)$. By the definition of $\mu_{\phi}$ it is clear that, for every $U \in C O(K)$ and for every $T \in \mu_{\phi}(U)$ one has $U=\mathrm{Cl}_{\phi X}(T) \cap K$. Then $z \in \bigcap\left\{U \mid \mu_{\phi}(U) \in G\right\}=\{y\}$, that is, $z=y$.

Theorem 3.8. Let $X$ be a space. Then $g_{X}$ is injective if and only if $\mathscr{L}^{\prime}(X)=$ $\mathscr{E}(X)$.

Proof. We use notation as in the above lemma. If $\mathscr{E}(X)=\mathscr{L}^{\prime}(X)$, that is, the inclusion map $j$ is an isomorphism, then $j^{*}$ is a homeomorphism, so that $t$ is also a homeomorphism. Thus, by the above lemma, $g_{X}$ is injective.

Now, suppose $g_{X}$ is injective. Then $\beta X \backslash X$ is 0 -dimensional, so that $q$ is the identity map. Then $\mu_{\phi}^{*} \circ t$ is a homeomorphism and $t$ is also a homeomorphism. Therefore $j^{*}$ is a homeomorphism, so that the inclusion map $j$ is bijective. This completes the proof.

Remark. It is easy to see that $\mathscr{L}^{\prime}(X)=\mathscr{E}(X)$ if and only if, for every open set $U \subset X, \bar{U}$ has compact boundary. This is also equivalent to the condition that every regular open set has compact boundary. In particular, this is true for every extremally disconnected space. Hence, the condition $\mathscr{L}^{\prime}(X)=\mathscr{E}(X)$ is intermediate between $\beta X \backslash X$ being 0 -dimensional and $X$ being extremally disconnected.

Proposition 3.9. If $X$ is normal and satisfies condition (C) then $l_{X}$ (equivalently, $\left.g_{X}\right)$ is irreducible.

Proof. Let $A$ be a proper closed subset of $\beta E X \backslash E X$. Then there is a nonempty subset of the form $h(V) \cap(\beta E X \backslash E X)$ which does not meet $A$. Then $V$ is not relatively compact (otherwise every element of $h(V)$ would be convergent). Put $W=\beta X \backslash \mathrm{Cl}_{\beta X}(X \backslash V)$. Then $W \cap X=V$ and $W \cap K \neq \varnothing$. In fact 
$W \cap K=\psi^{-1}([V])$, where $\psi$ is the isomorphism defined in the proof of Proposition 2.5. Let $y \in W \cap K$. For each $\mathscr{F} \in \beta E X \backslash E X$ converging to $y$, one has, clearly, $V \in \mathscr{F}$, that is, $\mathscr{F} \in h(V)$. Therefore $y \notin l_{X}(A)$.

\section{BIBLIOGRAPHY}

1. G. Birkhoff, Lectures on lattice theory, Amer. Math. Soc., Providence, RI, 1960.

2. G. L. Cain, Jr., R. E. Chandler, and G. D. Faulkner, Singular sets and remainders, Trans. Amer. Math. Soc. 268 (1981), 161-171.

3. A. Caterino, G. D. Faulkner, and M. C. Vipera, Construction of compactifications using essential semilattice homomorphism, Proc. Amer. Math. Soc. 116 (1992), 851-860.

4. W. W. Comfort and S. Negrepontis, The theory of ultrafilters, Springer-Verlag, 1974.

5. M. Katětov, On H-closed extensions of topological spaces, Časopis Pěst Mat. Frys. 72 (1947), 17-32.

6. K. D. Magill, The lattice of compactifications of a locally compact space, Proc. London Math. Soc. 18 (1968), 231-244.

7. J. Porter and R. G. Woods, Extensions and absolutes of Hausdorff spaces, Springer-Verlag, New York, 1988.

Department of Mathematics, North Carolina State University, Raleigh, North CarOLINA 27695-8205

E-mail address: gdf Omath.ncsu.edu

Dipartimento di Matematica, Università di Perugia, via Vanvitelli 1-06123 Perugia, ITALY

E-mail address: catvip@ipguniv.unipg.it 